\title{
Téoros
}

Revue de recherche en tourisme

\section{De l'importance des dépenses des spectateurs étrangers dans l'impact touristique des grands événements sportifs}

\section{Eric Barget et Jean-Jacques Gouguet}

Volume 30, numéro 2, 2011

URI : https://id.erudit.org/iderudit/1012247ar

DOI : https://doi.org/10.7202/1012247ar

Aller au sommaire du numéro

Éditeur(s)

Université du Québec à Montréal

ISSN

0712-8657 (imprimé)

1923-2705 (numérique)

Découvrir la revue

Citer cet article

Barget, E. \& Gouguet, J.-J. (2011). De l'importance des dépenses des spectateurs étrangers dans l'impact touristique des grands événements sportifs. Téoros, 30(2), 105-119. https://doi.org/10.7202/1012247ar

\section{Résumé de l'article}

De nombreuses études d'impact économique ont été commanditées afin de vérifier que les grands événements sportifs peuvent influer sur le niveau d'activité économique du territoire hôte. L'attraction de nombreux touristes est le plus souvent placée au centre de l'analyse. Néanmoins, ces travaux font toujours l'objet de multiples controverses. En effet, de redoutables problèmes méthodologiques se posent pour effectuer un tel calcul, et les résultats doivent être interprétés avec beaucoup de précautions. Nous avons essayé d'éviter les principaux biais liés au calcul d'impact pour élaborer une évaluation la plus fiable possible d'un méga-événement sportif. En utilisant la théorie de la base et en collectant une information de qualité sur le terrain, nous nous sommes efforcés d'éviter les erreurs les plus communes.

Nous avons choisi la Coupe du monde de rugby 2007, en France, pour illustrer ce débat. Les spectateurs étrangers sont à l'origine de l'essentiel du montant de l'impact économique sur les régions françaises, avec de grandes différences selon leurs nationalités et leurs profils sociodémographiques. Il apparaît ainsi que l'impact régional de la manifestation est d'autant plus fort que les parties accueillies ont attiré beaucoup de spectateurs étrangers, dotés d'un haut niveau de revenus et de dépenses. Des enseignements peuvent être tirés d'un tel résultat pour les organisateurs qui veulent accueillir un grand événement sportif. 


\title{
De l'importance des dépenses des spectateurs étrangers dans I'impact touristique des grands événements sportifs
}

\author{
Eric BARGET \\ Maître de conférences \\ CDES-OMIJ, Université de Limoges \\ eric.barget@unilim.fr \\ Jean-Jacques GOUGUET \\ Professeur des universités \\ CDES-OMIJ, Université de Limoges \\ gouguet@cdes.fr
}

\begin{abstract}
RÉSUMÉ: De nombreuses études d'impact économique ont été commanditées afin de vérifier que les grands événements sportifs peuvent influer sur le niveau d'activité économique du territoire hôte. L'attraction de nombreux touristes est le plus souvent placée au centre de l'analyse. Néanmoins, ces travaux font toujours l'objet de multiples controverses. En effet, de redoutables problèmes méthodologiques se posent pour effectuer un tel calcul, et les résultats doivent être interprétés avec beaucoup de précautions. Nous avons essayé d'éviter les principaux biais liés au calcul d'impact pour élaborer une évaluation la plus fiable possible d'un méga-événement sportif. En utilisant la théorie de la base et en collectant une information de qualité sur le terrain, nous nous sommes efforcés d'éviter les erreurs les plus communes.

Nous avons choisi la Coupe du monde de rugby 2007, en France, pour illustrer ce débat. Les spectateurs étrangers sont à l'origine de l'essentiel du montant de l'impact économique sur les régions françaises, avec de grandes différences selon leurs nationalités et leurs profils sociodémographiques. II apparaît ainsi que l'impact régional de la manifestation est d'autant plus fort que les parties accueillies ont attiré beaucoup de spectateurs étrangers, dotés d'un haut niveau de revenus et de dépenses. Des enseignements peuvent être tirés d'un tel résultat pour les organisateurs qui veulent accueillir un grand événement sportif.
\end{abstract}

Mots-clés: Grands événements sportifs, impact économique, retombées touristiques, profil de dépenses, théorie de la base.

On considère généralement que les grands événements sportifs peuvent générer un impact économique considérable sur les territoires d'accueil, grâce en particulier à l'arrivée de nombreux touristes. Néanmoins, l'analyse économique d'un tel impact fait toujours l'objet de multiples débats, d'une part du fait de la très grande variabilité des résultats obtenus selon les évaluateurs, d'autre part par rapport à l'utilisation politique de tels résultats. La plupart du temps, dans la phase de préparation de l'accueil d'un grand événement sportif, on constate la prééminence du calcul de retombées économiques pour justifier la décision. Or, il existe un consensus dans la communauté scientifique des économistes du sport pour reconnaître que la légitimité de l'organisation d'un événement ne peut pas reposer sur le calcul d'impact économique à lui seul (Kesenne, 2005; Baade, 1996; Martin, 1991). Il faut pour cela également un calcul de rentabilité sociale (coûts/bénéfices).

Deux calculs doivent donc être menés : un calcul de retombées économiques pour mesurer les effets d'entraînement du spectacle sportif sur le territoire; un calcul d'utilité sociale qui consiste à mesurer le bénéfice social net ou la perte sociale nette généré par l'événement. Ces deux calculs sont complémentaires : l'analyse coûts/bénéfices apporte une réponse à un problème qui n'était pas envisagé par le calcul d'impact économique, et vice versa. L'étude d'impact privilégie les visiteurs et financeurs extérieurs au territoire d'accueil (théorie de la base); l'analyse coûts/bénéfices met l'accent sur le bien-être des habitants du territoire d'accueil. Ces deux calculs se complètent pour l'amélioration de la prise de décisions. En effet, les objectifs du décideur peuvent être multiples : amélioration du bien-être de sa population, développement économique, amélioration de l'image de marque, de l'identité, de l'attractivité de son territoire.

Dans le cadre du présent article, nous en resterons au calcul de l'impact à court terme d'un grand événement sportif : la Coupe du monde de rugby 2007, en France (ou Rugby World Cup/RWC). Au-delà des résultats très optimistes 
auxquels aboutissent les études menées avant l'événement (ESSEC, 2007; MKG Hospitality, 2007), il apparait important de réfléchir à leurs conditions de validité. Voilà pourquoi, à la place d'une étude ex ante toujours très fragile, nous avons préféré mener une analyse ex post de la Coupe du monde de rugby pour évaluer notamment son impact touristique. La Coupe du monde de rugby constitue un événement atypique. Elle peut en effet être considérée comme un grand événement sportif au niveau planétaire compte tenu de sa médiatisation et de son audience (voir encadré 1). Pourtant, ses modalités d'organisation (son budget nettement plus faible que celui des plus grands événements sportifs, le caractère limité des investissements, etc.) ainsi que la forte concentration sur quelques pays de l'engouement pour le rugby au plan international tendent à atténuer la place de la Coupe du monde de rugby en tant que méga-événement.

La Coupe du monde de rugby pose une difficulté d'analyse spécifique aux événements multi-sites. Elle a été accueillie par

\section{ENCADRÉ 1: LES CHIFFRES CLÉS DE LA COUPE DU MONDE DE RUGBY 2007, EN FRANCE}

\section{Cadrage général :}

Nombre de rencontres : 48 (dont 2 en Écosse et 4 au Pays de Galles)

Nombre d'équipes : 20

Nombre de joueurs : 600

Nombre de volontaires : 6000

\section{Spectateurs :}

Billets vendus : 2,25 millions

Taux de remplissage des stades : 95,4\%

Télévisions :

Nombre de pays diffuseurs : 200

Heures de couverture TV : 6500

Audience cumulée : 4 milliards de spectateurs

Audience nationale record pour France vs Angleterre (demi-finale) :

18,3 millions de spectateurs

\section{Budget d'organisation :}

Budget du GIP : 255 M d'euros

Bénéfice net avant impôt pour le GIP : 33 M d'euros

Droits perçus par l'IRB :

Droits TV :

- Montant des droits TV : 130 M d'euros dont 70 M d'euros versés par TF1 pour les éditions 2007 et 2011

Partenariat :

- Tickets d'entrée des partenaires officiels : $5 \mathrm{M}$ d'euros

- Tickets d'entrée des sponsors officiels : 2,5 M d'euros

Produits dérivés :

- Nombre de sociétés ayant obtenu des licences de l'IRB : 35

- Nombre de références de produits dérivés CMR : 700

- Points de vente provisoires dans les villes hôtes : 80

Ensemble des droits médias et marketing :

- Ensemble des droits médias et marketing commercialisés

par l'IRB : 190 M d'euros

Animation :

Actions d'animation dans toute la France : 1000

Source : compilation des auteurs. dix villes et huit régions françaises bénéficiant ainsi de retombées économiques qu'il est intéressant d'analyser tant dans leur ampleur que dans leur structure pour faire apparaitre des spécificités régionales :

- quels sont les facteurs explicatifs des différences régionales dans l'ampleur de l'impact économique de la Coupe du monde de rugby?;

- quel est, globalement, la place des dépenses des touristes étrangers?;

- assiste-t-on à des différences significatives selon la nationalité des touristes?

Pour répondre à toutes ces questions, la seconde section présente, au travers d'une revue de la littérature, le cadre méthodologique de la mesure de l'impact touristique des grands événements sportifs. Une troisième section présente les conditions de mise en œuvre du calcul d'impact sur le terrain. Une quatrième section commente et discute les résultats obtenus. Une cinquième section conclut.

\section{Cadre méthodologique \\ Deux approches : rentabilité sociale et impact économique}

Il existe deux grands types de méthodes pour appréhender les effets d'un projet en général, d'un événement sportif en particulier, sur le territoire : le calcul de rentabilité sociale (Barget \& Gouguet, 2010a) d'une part, l'impact économique (Barget \& Gouguet, 2010b) d'autre part. Nous allons présenter les principales caractéristiques de ces méthodes qui reposent sur des paradigmes différents (théorie du bien-être pour l'une, théorie du développement économique régional pour l'autre) et ne visent pas à mesurer le même type d'effets économiques.

\section{Rentabilité sociale}

La théorie du bien-être (Mankiw, 1998) propose plusieurs instruments d'évaluation de projets permettant d'apprécier la variation du bien-être collectif qui en résultera. Tous ces outils sont issus de la Welfare Economics et constituent le calcul économique (Perret, 2004). Il s'agit de mettre en relation les moyens mobilisés par un projet (coûts) et les résultats obtenus (bénéfices), et sur cette base d'effectuer des choix entre projets alternatifs. Dans un monde de relative rareté des ressources, le choix des décideurs publics dans l'allocation des ressources communes peut être éclairé par l'économiste, sur la base de l'accroissement de satisfaction que procurent les différents projets à la communauté concernée. L'approche est normative puisqu'elle vise à aider les décideurs publics à faire des choix dans l'usage des deniers publics. La notion de coût d'opportunité est centrale, l'argent utilisé pour un projet n'est plus disponible pour un autre; les bénéfices qu'aurait générés ce projet alternatif auquel il a fallu renoncer ont été perdus, ils constituent le coût d'opportunité du projet retenu (Preuss, 2007a).

Les méthodes procèdent toutes de la même manière, néanmoins elles ne vont pas toutes aussi loin dans la prise en compte, et la valorisation, des bénéfices et des coûts des projets publics. Le bilan des coûts et des bénéfices associés à chacun des projets suit des procédures et revêt des formes diverses : l'analyse coûts-efficacité, l'analyse coûts-utilité et 
l'analyse coûts-avantages. La distinction entre ces approches se focalise autour de la question de la monétarisation des bénéfices et des coûts. L'analyse coûts-avantages va plus loin que les analyses coûts-efficacité et coûts-utilité, en permettant le calcul du gain ou de la perte monétaire nette pour la communauté. Cette approche procède, en effet, à la monétarisation de l'ensemble des bénéfices et des coûts des projets concurrents. Il est possible, à partir de cette méthode, de réaliser une classification des projets selon qu'ils sont plus ou moins désirables pour la société, à partir du critère du bénéfice social net ou de la perte sociale nette (différence entre les bénéfices et les coûts). L'analyse coûts-efficacité appréhende les conséquences des projets en termes d'unités physiques (les plus appropriées possibles) : un ensemble prédéfini d'objectifs est spécifié. En effet, de deux projets, on choisira celui qui permet de parvenir à ces objectifs aux moindres coûts. Il s'agit d'atteindre une efficacité donnée en minimisant les coûts ou, inversement, d'atteindre une efficacité maximale pour un coût donné. L'analyse coût-utilité ne se contente pas d'appréhender les résultats du projet en termes d'unités physiques, mais on affecte des «utilités » à ces unités physiques. Dans le cadre de la Coupe du monde de rugby, nous avions retenu l'analyse coûts-avantages, plus aboutie, de notre point de vue. Les résultats ont été publiés par ailleurs (Barget et Gouguet, 2010d); ils ne sont pas repris ici.

\section{Impact économique}

L'étude d'impact économique constitue une application des théories du développement économique régional, en ce sens qu'elle tente de quantifier les conséquences économiques d'une activité permanente (une base militaire, un équipement sportif, etc.) ou temporaire (un festival, un événement sportif, etc.) sur un territoire déterminé (Jeanrenaud, 1999). Elle contribue ainsi à expliquer les inégalités de développement territorial. C'est en raison de cette opérationnalité de la méthode que le calcul d'impact économique a connu un succès considérable au cours des 40 dernières années. En ce qui concerne directement les événements sportifs, l'objectif général est de montrer comment ils influent sur l'activité économique du territoire hôte en mobilisant des agrégats tels que la valeur ajoutée, l'emploi ou les rentrées fiscales supplémentaires.

Il n'existe pas de calcul d'impact économique standard, les travaux en matière sportive comme ailleurs étant particulièrement hétérogènes. Des angles d'observation divergents sont retenus d'une étude à l'autre. Le chercheur peut tenter de rendre compte de la modification des agrégats économiques intervenant sur la courte période (phase de déroulement de l'événement) ou bien, au contraire, sur le long terme (phase de préparation et période postérieure à la manifestation), ce qui pose la question du choix de l'horizon temporel retenu. Il peut procéder à l'évaluation des effets à des niveaux spatiaux différents allant de la localité jusqu'à l'État, ce qui a conduit à réfléchir à la notion d'espace pertinent pour le calcul d'impact (Dion, 1987). Outre le choix de l'agrégat et du territoire d'analyse, le chercheur peut s'intéresser à la génération de revenus additionnels ou à la redistribution de ces revenus à l'intérieur du territoire. La grande diversité des motivations et des angles d'observation sous-jacents aux études d'impact économique explique la multiplicité des modèles mobilisés, ceux-ci allant de l'analyse économétrique à l'usage de modèles macro-économiques sophistiqués, en passant par l'emploi de multiplicateurs plus ou moins désagrégés. C'est cette dernière approche, d'ailleurs la plus fréquente, que nous retiendrons dans le cadre de cet article. Sur la base d'une injection qui correspond aux effets directs et indirects, les effets induits peuvent être calculés par application d'un coefficient multiplicateur qui traduit les rondes successives de dépenses et de revenus dans l'espace local.

La recherche de points communs entre les études trouve donc très rapidement ses limites, les économistes n'appréhendent le spectacle sportif qu'à travers quelques-uns de ses bénéfices et ne parviennent qu'à avoir une vision incomplète de ce phénomène économique. Ce constat a été effectué par Davidson (1999), qui l'exprime de manière imagée. Ainsi, l'économiste en charge d'une étude d'impact serait comme une fourmi sur le dos d'un éléphant. En fonction $\mathrm{du}$ point d'observation retenu, il pourra scientifiquement déduire des conclusions très différentes sur l'objet d'étude, sans jamais parvenir à en avoir une représentation complète. Par ailleurs, les études d'impact économique ne considèrent que les bénéfices et ignorent les coûts, voire, pour certains (Stringer, 1993), transforment les coûts en bénéfices. Cela constitue une différence majeure avec l'analyse coûts-avantages, il en résulte un risque potentiel de biais en faveurs des grands projets très couteux. L'étude d'impact ne peut dans ces conditions constituer à elle seule un instrument permettant de légitimer la décision d'accueillir la manifestation (Jeanrenaud, 1999; Barget et Gouguet, 2010c). De ce point de vue, la mesure des retombées économiques des événements sportifs constitue une thématique particulièrement controversée. Des voies s'élèvent au sein de la communauté scientifique (économistes, statisticiens, etc.) pour dire qu'il s'agit d'études-alibis donnant systématiquement des retombées économiques importantes et intégrant des erreurs de méthode conduisant à des résultats bien souvent largement surévalués (Barget et Gouguet, 2010a).

Les résultats en matière de retombées économiques sont le plus souvent retenus dans la prise de décision, et on constate une forte demande sociale de ce type d'études. Cela s'explique par le fait que les emplois et les revenus créés constituent un argument essentiel d'acceptation du projet (et des coûts inhérents) par la population. L'accent est fréquemment mis sur les enjeux pour l'industrie touristique, ce qui n'est pas étonnant puisque, si les événements donnent lieu à des investissements et à des frais d'organisation variables (parfois limités), ils occasionnent systématiquement des déplacements massifs de supporteurs. L'hypothèse selon laquelle les spectacles sportifs pourraient entraîner l'économie touristique est largement retenue par les décideurs politiques qui se lancent dans de véritables stratégies d'accueil systématique d'événements sportifs dont le développement du tourisme est un objectif majeur (Chappelet, 2006; Teed, 2006). L'une des caractéristiques essentielle des spectacles sportifs, qui a d'ailleurs été largement utilisée par les économistes pour définir la notion d'événement sportif exceptionnel ou «hallmark event» (Ritchie et Yangzhou, 1987), consiste en leur 
forte capacité d'attraction. L'impact touristique peut ainsi se définir comme la résultante des dépenses des visiteurs (supporteurs assistant à l'événement et leurs accompagnateurs, des sportifs et leur encadrement, des officiels et des VIP). Nous traiterons ici de l'impact touristique de la Coupe du monde de rugby 2007, en phase avec la véritable culture du calcul d'impact qui existe dans le secteur du tourisme (Madden, 2002; Preuss, 2007b; Li et Blake, 2009). Par comparaison, lorsqu'on évoque l'impact économique, on entend les retombées liées aux dépenses des visiteurs, mais également des frais d'organisation (comité organisateur) et des investissements (pour l'essentiel publics).

\section{Les instruments du calcul d'impact}

Deux types de modèles semblent s'imposer au cours des dernières années pour effectuer le calcul d'impact touristique des grands événements sportifs : le modèle input-output et les modèles d'équilibre général calculable (Kasimati, 2003). Devant les difficultés liées à leur mise en ouvre opérationnelle, nous avons opté pour le modèle de la base économique plus adapté au cas français.

\section{Input-output}

Le multiplicateur de Leontief (ou input-output) constitue une approche entièrement désagrégée qui permet de cerner les effets d'entraînement d'un choc initial de demande sur les différents secteurs de l'économie. Le caractère désagrégé de la méthode la rend particulièrement propice pour l'analyse d'activités spécifiques, telles que les spectacles sportifs ponctuels qui ont des effets prononcés sur le secteur du BTP (pour ce qui est des investissements) et du tourisme (dépenses des supporteurs). La structure économique régionale doit préalablement être reconstituée de manière complète par l'identification des échanges interindustriels et la constitution d'un tableau entrées-sorties : autant de secteurs que les données le permettent sont distingués. Pour chacun d'eux, il faut établir quels sont les inputs utilisés dans le processus de production et déterminer de quelle manière les extrants (la production) vont se répartir entre consommation intermédiaire des entreprises et consommation finale des individus. À partir de la connaissance de ces échanges interindustriels, il est possible d'estimer la variation sectorielle totale de la production nécessaire pour satisfaire la demande initialement née dans un seul d'entre eux (Blake, 2005).

Cette approche est séduisante par son caractère désagrégé, d'autant qu'il est également possible de ventiler les retombées quant à leur répartition spatiale, ou selon les professions et catégories sociales des bénéficiaires. Cependant le modèle est très exigeant en informations puisque l'ensemble des relations interindustrielles et des échanges interrégionaux doit être connu. Il constitue donc un choix pertinent pour les pays ayant un niveau d'avancement important des statistiques économiques régionales, principalement les pays anglo-saxons (Porter et Fletcher, 2008; Dwyer et al., 2003). Dans les autres pays, on s'en remet davantage à des multiplicateurs ou modèles macro-économiques régionaux, la théorie de la base ou les multiplicateurs de type keynésiens étant en général retenus.

\section{Méthode d'équilibre général calculable}

Comme le rappelle Blake (2005) les modèles d'équilibre général calculable (MEGC) ont été utilisés dans une grande variété de domaines allant du commerce international aux politiques agricoles, de développement économique et de protection de l'environnement, et plus récemment dans le secteur touristique. Ces modèles intègrent des relations clés qui ne sont pas prises en compte dans les modèles input-output (figure I-29, dans Blake, 2005). Ils connaissent un succès croissant pour les études d'impact économique d'événements sportifs, ce qui peut s'expliquer par le fait qu'il s'agit de sophistications du modèle input-output.

Le modèle calculable d'équilibre général introduit en plus les effets sur les prix qui sont supposés constants dans l'approche input-output, alors qu'une accélération de l'inflation est inhérente aux grands événements sportifs. Rappelons en outre, que les MEGC imposent des contraintes sur la disponibilité des facteurs de production (travail et capital), de sorte que, selon le scénario choisi, l'offre de ces facteurs est soit fixe soit variable; l'offre de facteurs répond à des variations de prix plutôt que de postuler leur disponibilité en quantité illimitée pour satisfaire la demande. L'offre de facteurs de production n'est donc plus supposée être parfaitement élastique. Là où le modèle input-output ajoute les effets direct, indirects et induits du tourisme et débouche sur un multiplicateur du tourisme en général supérieur à un, le MEGC intègre les déplacements de ressources qui peuvent intervenir entre le tourisme et les autres secteurs économiques. Il peut y avoir diminution de la production des autres secteurs. Les modèles MEGC intègrent le fait que les ressources (inputs ou consommations intermédiaires) peuvent simplement être déplacées des autres secteurs de l'économie vers le tourisme (Copeland, 1991). Contrairement à l'approche input-output, le résultat de l'étude peut être négatif dans certains secteurs.

Ainsi, les MEGC sont supposés donner des résultats plus réalistes, pourtant leur validité dépend des hypothèses fortes posées dans le modèle (U.R.S. Finance and Economics, 2004; Blake, 2005). Il peut, par ailleurs, exister un décalage entre la sophistication de ces modèles et la réalité locale, ce qui nous a conduits à leur préférer la théorie de la base économique.

\section{Théorie de la base}

Quand on parle de base économique, on cherche généralement à déterminer les potentialités économiques d'un territoire qui sont les plus fondamentales pour le développement de ces unités (Gouguet, 1981; Davezies, 2008). Ce sont les activités motrices, les autres étant induites. On a donc ici une théorie dichotomique de la croissance du fait de cette reconnaissance de deux types d'activités bien distinctes. Le spectacle sportif peut être considéré comme une activité basique (Bourg et Gouguet, 1998). Cette théorie semble particulièrement adaptée aux territoires de petite dimension comme ceux de la Coupe du monde de rugby. Les grands événements sportifs, considérés comme activités basiques, constituent un moteur de la croissance économique de ces espaces. En effet, ils font rentrer de l'argent frais dans les territoires concernés et, par effet de multiplication, vont engendrer une création de valeur ajoutée et d'emploi (voir illustration 1). 


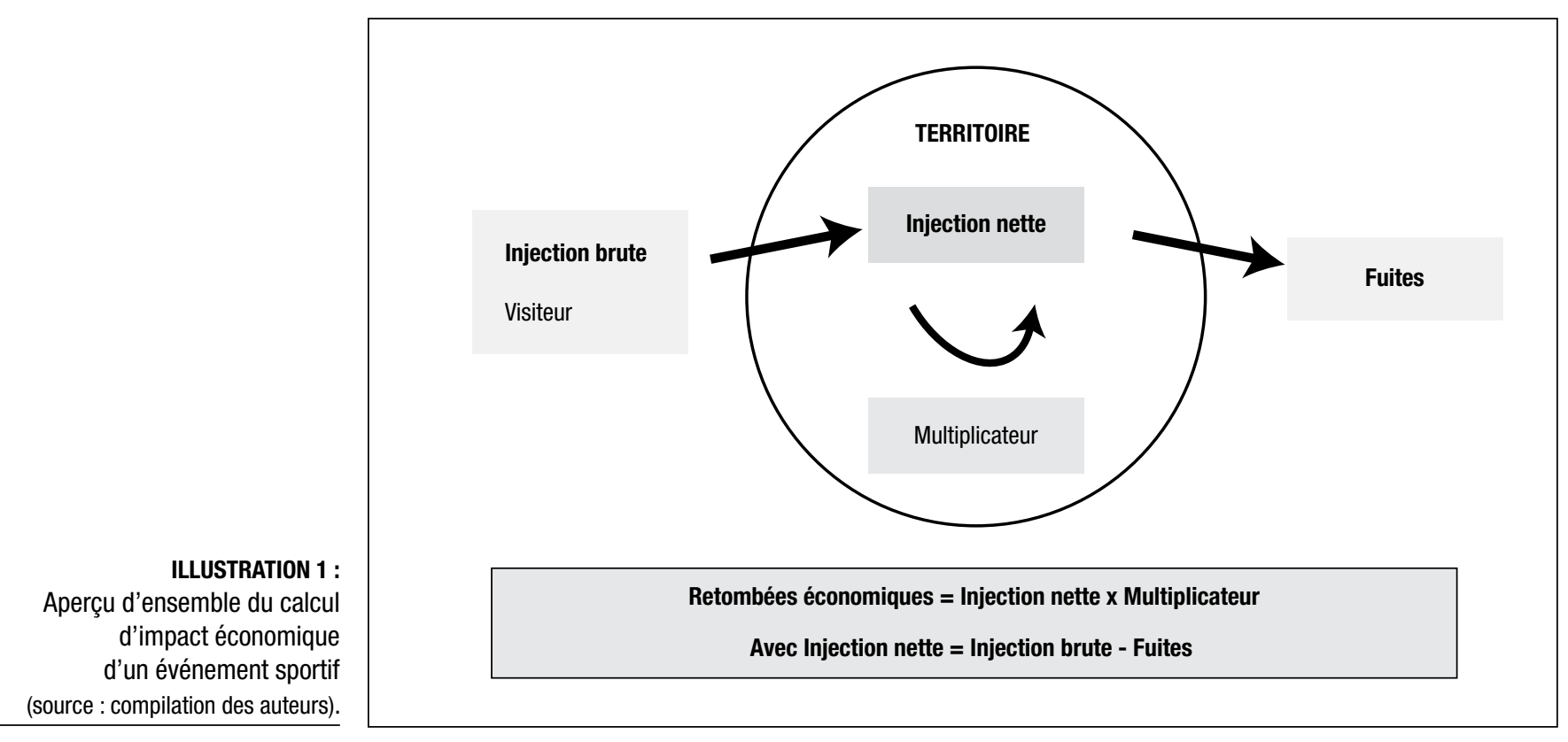

Néanmoins, si la théorie de la base est relativement simple dans son principe, elle présente des difficultés dans sa mise en œuvre qui, si on n'y prend pas garde, peuvent conduire à des erreurs. Sur les petits territoires, l'ampleur de l'impact va dépendre de deux éléments : la dimension du projet et le degré d'intégration du territoire autour de sa structure productive.

La dimension du projet conditionne souvent le recours à des opérateurs spécialisés extérieurs. De gros projets sur des petits territoires profitent souvent ainsi à l'extérieur (voir l'exemple des Jeux olympiques d'hiver d'Albertville). Quant au degré d'intégration du territoire autour de sa structure productive, plus le degré d'intégration est fort et plus les fuites sont faibles. Plus la région est petite, plus elle est peu diversifiée et intégrée. Plus les fuites risquent d'être importantes, plus le multiplicateur va être faible.

Par rapport au calcul d'un tel multiplicateur, les chercheurs anglo-saxons sont favorisés par le degré d'aboutissement de leur système de comptabilité économique régionale. Les statistiques existant en matière de relations interindustrielles et d'échanges interrégionaux permettent la construction de matrices input-output régionales puis la simulation désagrégée de l'impact régional. En France, rien de tel n'existe, et l'on doit se contenter de calculer des multiplicateurs agrégés de revenus, dans la mesure où des multiplicateurs désagrégés de dépenses selon les catégories d'agents impliqués demanderaient des enquêtes de terrain longues et coûteuses. Ici, il était souhaitable de calculer un coefficient multiplicateur sur les mêmes bases pour toutes les huit régions hôtes, ceci à des fins de comparabilité et d'équité dans le traitement. Plus précisément, le coefficient multiplicateur a été calculé à partir de la méta-analyse proposée par Vollet et Bousset (2002) dont nous avons repris l'estimation suivante :

(5) $\mathrm{k}=-0,53+0,17$ In $P O P-0,025$ In $P R I+0,083$ In TER
La relation positive entre la taille de la population $(P O P)$ et la valeur du multiplicateur exprime l'influence du degré de diversification de la structure régionale. On peut faire l'hypothèse selon laquelle plus la région est grande (et diversifiée), plus la propension à importer sera faible et la propension à dépenser local, forte, d'où une valeur élevée du multiplicateur.

L'impact de la structure de la population active sur la valeur du multiplicateur confirme les analyses généralement effectuées : toutes choses égales par ailleurs, le multiplicateur sera d'autant plus élevé que la proportion d'emploi tertiaire $(T E R)$ est forte et inversement par rapport à la proportion d'emploi primaire (PRI).

\section{Mise en œuvre de la recherche} Les erreurs à éviter

Trop souvent, les études de retombées économiques de grands événements sportifs surévaluent considérablement les résultats, du fait d'erreurs de calcul liées à une méthodologie insuffisamment rigoureuse (Preuss, 2006). Nous avons cherché à éviter les plus courantes, soit :

a) la non-prise en compte de l'effet de substitution qui concerne les dépenses des agents locaux, dépenses qui doivent donc être exclues. Si la Coupe du monde de rugby n'avait pas eu lieu, les agents locaux auraient certainement effectué des dépenses d'une autre nature sur le territoire, d'où une absence d'impact. Cela explique la seule prise en compte des dépenses des agents extérieurs à la région pour la détermination de l'injection nette.

b) la non-prise en compte du déplacement temporel de dépenses de consommation ou d'investissement. Cela concerne les décisions prises par les agents extérieurs avant la Coupe du monde de rugby et qui ont été simplement déplacées (avancées ou repoussées) pour se réaliser durant l'événement. On ne peut alors attribuer à la Coupe du monde de rugby l'augmentation du niveau d'activité correspondant. 
Notre questionnaire permet de repérer les individus qui ont déplacé leur visite. De même nous avons pu repérer les visiteurs occasionnels dont la présence sur les lieux de la manifestation n'est pas due à la Coupe du monde de rugby mais à des motifs professionnels, par exemple. S'ils assistent à la rencontre, seule la dépense sportive additionnelle doit être prise en compte.

c) l'omission des effets d'éviction en matière de consommation ou d'investissement. Des visiteurs potentiels peuvent avoir été découragés de venir dans la région du fait de la crainte de la saturation du territoire ou de nuisances créées par la Coupe du monde de rugby. Nous avons élaboré un protocole de mesure de tels effets d'éviction sur la base de la comparaison des statistiques hôtelières septembre-octobre 2007 et septembre-octobre 2006. Un tel calcul n'a pas été fait systématiquement dans les régions d'accueil, nos entretiens approfondis nous ayant révélé que ces effets d'éviction étaient, en l'espèce, très faibles par rapport à d'autres grands évènements sportifs mondiaux.

d) l'omission des fuites hors territoire (consommation ou investissement), source de surévaluation de l'impact quand on retient le montant brut de la dépense au lieu de la valeur ajoutée qui permet de prendre les fuites dues aux consommations intermédiaires ou aux produits importés. Il en est de même des doubles comptes (voir illustration 2) qui peuvent intervenir à l'intérieur de l'injection touristique (un spectateur «écran géant» ou "stade», ou un accompagnateur peut parfois ne constituer qu'une seule et même personne, la dépense ne devant être considérée qu'une seule fois) et à l'intersection entre l'injection relative aux dépenses d'organisation (les achats de billets constituent la recette majeure du comité d'organisation, et ils ne devront pas être comptés une seconde fois dans les dépenses des visiteurs).

La surévaluation du multiplicateur est fréquente, certains utilisant des multiplicateurs obtenus dans diverses études et sortis de leur contexte territorial. Pour éviter ce biais, nous avons préféré utiliser le même calcul pour toutes les régions à des fins d'homogénéité des résultats. Sur les bases expliquées précédemment, la valeur du multiplicateur oscille entre 2,02 (Languedoc-Roussillon) et 2,35 (Île-de-France). Ces résultats semblent légèrement plus élevés que ceux d'études ponctuelles réalisées (voir par exemple Doucet, 2002). De toute façon, il apparaît que, au delà de telles différences relativement minimes, l'essentiel se joue au niveau du calcul des injections nettes, ce qui implique la collecte d'une information fiable sur le terrain.

\section{L'enquête de terrain}

Rappelons que les effets d'entraînement d'un grand événement sportif sur un territoire proviennent de trois types de dépenses :

a) les dépenses de consommation;

b) les dépenses liées à l'organisation et à l'animation;

c) les dépenses d'investissement.

Les dépenses de consommation sont le fait des spectateurs "stades», des spectateurs "écrans géants", des équipes nationales et des accompagnateurs de ces différents agents économiques. On ne retient que les consommateurs extérieurs à la région (reste de la France et étrangers), ce qui permet de comprendre une partie des différences territoriales dans l'impact de la Coupe du monde de rugby 2007, le pourcentage d'étrangers et de spectateurs hors région variant selon les cas.

Les dépenses liées à l'organisation et à l'animation reflètent la spécificité de l'organigramme de la Coupe du monde de rugby 2007. Un Groupement d'Intérêt Public a été créé à Paris pour superviser l'organisation générale; un comité local de coordination a été installé dans chaque région pour élaborer et mettre en œuvre les actions de valorisation. Surtout, la structure de financement est très spécifique, avec des fuites proportionnellement plus élevées que dans des compétitions comme les JO ou la Coupe du monde de football. Ainsi, l'IRB (International Rugby Board), propriétaire de la Coupe du monde de rugby et dont le siège est en Irlande, perçoit directement l'essentiel des recettes commerciales de l'événement (commandite, droits de télédiffusion, VIP, loges, pour un total de $130 \mathrm{M}$ d'euros de droits médias et de $60 \mathrm{M}$ d'euros de commandites). Le modèle économique du Groupement d'Intérêt Public repose ainsi presque exclusivement sur les recettes de billetterie.

Les dépenses d'investissement concernent la construction et la rénovation des stades, principalement. C'est l'État et les collectivités territoriales qui sont concernés ici pour le financement de telles opérations. Cependant, ces investissements sont restés très limités puisque l'événement a été organisé dans des stades préexistants, accueillant habituellement des rencontres de football d'une capacité supérieure aux stades du rugby français.

Au final, il s'agit de repérer parmi tous les flux précédents ceux qui sont issus d'agents extérieurs au territoire régional pour calculer les injections, de retrancher toutes les fuites hors territoire et d'appliquer le multiplicateur régional, la phase de collecte des données étant déterminante quant à la qualité de l'estimation. Pour cela, nous avons administré 5500 questionnaires remplis en face à face auprès des spectateurs (stades et écrans géants) et des accompagnateurs. Le questionnaire spectateur en particulier se structure de la façon suivante :

a) Une question filtre était d'abord posée sur l'âge de la personne interrogée, et l'entretien ne se poursuivait que dans le cas où elle avait au moins 15 ans. Le choix de descendre jusqu'à la limite de 15 ans permet de prendre en compte le fait que certains jeunes sont venus en groupe et non en famille; à cette occasion, ils ont réalisé des dépenses limitées, à titre personnel. Ignorer ce type de profils de spectateurs aurait conduit à surestimer la dépense moyenne d'un spectateur. En revanche, le risque de l'abaissement de la limite d'âges pour intégrer l'échantillon est que la somme annoncée par les jeunes corresponde à de l'argent de poche, ce qui pose problème lorsqu'ils sont venus en famille (nous aurions dans ce cas souhaité avoir la dépense de l'ensemble de la famille). Nous n'avons pas étudié les répercussions qu'aurait le fait de porter la limite à 18 ans, on peut néanmoins penser qu'elles seraient probablement mineures, sachant que pour le site de Bordeaux, par exemple, un groupe composé en moyenne de 4,3 personnes comprend 0,4 jeunes de moins de 18 ans. 


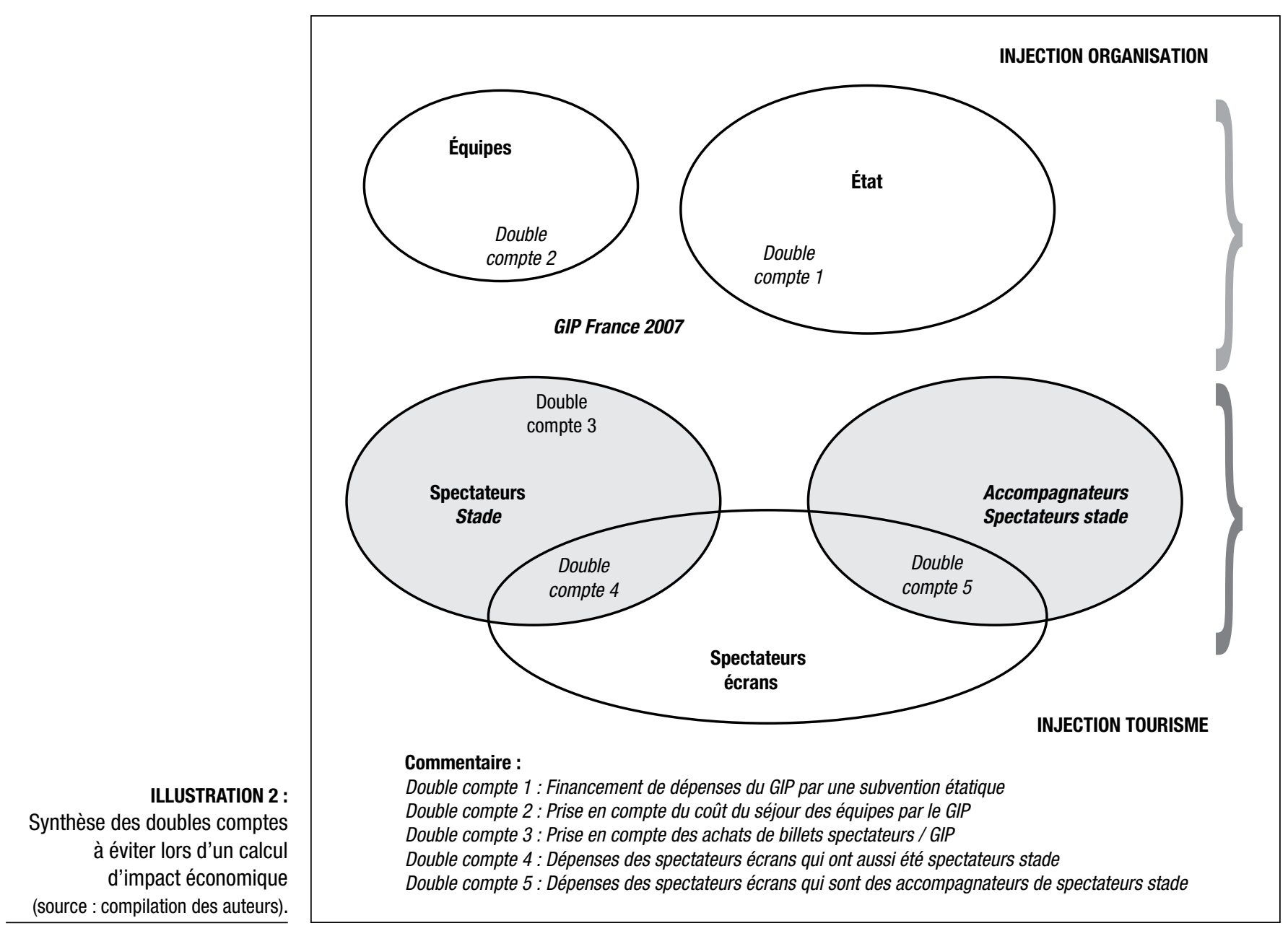

b) Des questions concernaient le lieu de résidence et les conditions du déplacement pour se rendre au stade (moyen de transport utilisé, distance parcourue, nombre de personnes dans le véhicule, etc.). Le lieu d'habitation est particulièrement important lors d'une étude d'impact, puisque, selon la théorie de la base économique, seuls les agents économiques extérieurs au territoire d'analyse (ici, le territoire de la région hôte de la Coupe du monde de rugby) peuvent être considérés comme source d'injection d'argent à travers leurs dépenses. Les autres données collectées sur les modalités du voyage, étaient davantage destinées au calcul de la valeur d'usage, dans le cadre de l'analyse coûtsavantages de la Coupe du monde de rugby.

c) Un second bloc portait sur les motifs et la programmation du voyage, celui-ci devant permettre d'identifier les individus pour lesquels la Coupe du monde de rugby avait été déterminante quant à la décision de se déplacer. Les réponses doivent servir à déterminer si les dépenses engagées localement par un spectateur extérieur peuvent être considérées comme étant réellement une résultante de la Coupe du monde de rugby ou si elles auraient eu lieu même sans elle. Il s'agit d'exclure les dépenses des visiteurs occasionnels (venus pour un autre motif et en profitant pour assister à la Coupe du monde) et les dépenses simplement déplacées dans le temps plutôt qu'additionnelles (si le voyage était programmé et a été avancé ou reculé pour le faire correspondre aux dates de la Coupe du monde de rugby).

d) Un passage sur la composition du groupe permettait de connaître le type d'individus qui accompagnent le spectateur. Ceci est d'importance, puisque la dépense qui est demandée ensuite porte sur l'ensemble des membres de la famille et qu'il faudra passer à la dépense individuelle par simple division. Il convient également d'identifier le nombre d'accompagnateurs qui n'entrent pas dans les stades, puisqu'il faudra également prendre en compte leurs dépenses (nécessairement hors stade), qui ne seraient pas engagées sans la Coupe du monde de rugby.

e) Le bloc portant sur le séjour comprenait une question sur le nombre de rencontres de la Coupe du monde vues, site par site. Le but est de connaître le nombre de rencontres vues dans la région. Il suffit ensuite de diviser la dépense par le nombre de parties pour obtenir une dépense par partie. D'autres questions traitent du nombre de nuitées passées hors domicile et du mode d'hébergement utilisé, ce qui est utilisé comme variable de contrôle de la dépense d'hébergement annoncée. 
f) Le passage suivant était fondamental, puisqu'on y demandait, poste par poste, le montant dépensé, en dissociant la partie profitant à la ville hôte, et celle bénéficiant à la région (déterminante puisque le territoire d'analyse est la région administrative). Les enquêteurs avaient pour consigne de consacrer davantage de temps à cette question sensible et impliquant plusieurs réponses à porter dans un tableau (voir encadré 2). Les dépenses des spectateurs locaux n'avaient pas d'intérêt pour le calcul d'impact, mais des comparaisons utiles ont pu être effectuées avec la structure de dépense des spectateurs extérieurs à la région (visiteurs), qui, eux, génèrent des retombées économiques.

g) La partie suivante traitait des caractéristiques personnelles du spectateur, avec à la fois des informations socioéconomiques très classiques (âge, sexe, diplôme, situation familiale, etc.) et des renseignements plus spécifiques au sport (licence sportive, nombre de spectacles sportifs vus, etc.), ou au rugby (licence, nombre de spectacles de rugby, etc.). Ces renseignements sont utiles pour mieux cerner le profil du public, appréhender les variables qui jouent sur leurs dépenses. Plus directement en relation avec le calcul d'impact économique, la tranche de revenu mensuel annoncée pour l'ensemble du ménage a permis d'affiner la dépense par catégorie de foyers (gagnant plus ou moins de $4000 €$ par mois).

L'entretien se terminait, après avoir identifié les catégories de billets possédés, puis les conditions de l'entretien

\section{ENCADRÉ 2: QUESTION 14 EXTRAITE DU QUESTIONNAIRE}

Quel montant aurez-vous dépensé pour vous et les membres de votre famille concernant les postes que je vais vous citer? Indiquez pour chacun de ces postes le pourcentage approximatif dépensé dans la ville et dans la région.

\begin{tabular}{|c|c|c|c|}
\hline & Montant & $\begin{array}{l}\% \text { dans } \\
\text { la ville }\end{array}$ & $\begin{array}{l}\% \text { dans } \\
\text { la région }\end{array}$ \\
\hline q14a Transports & & & \\
\hline q14b En logement & & & \\
\hline $\begin{array}{l}\text { q14c Dépenses dans le stade } \\
\text { (hors billet) }\end{array}$ & & & \\
\hline $\begin{array}{l}\text { q14d Nourriture hors du stade } \\
\text { (restaurant, alimentation) }\end{array}$ & & & \\
\hline $\begin{array}{l}\text { q14e Sorties/Divertissements } \\
\text { (cafés, cinéma, discothèque...) }\end{array}$ & & & \\
\hline q14f Tourisme & & & \\
\hline $\begin{array}{l}\text { q14g Services divers } \\
\text { (laverie, coiffeur, médecin...) }\end{array}$ & & & \\
\hline $\begin{array}{l}\text { q14h Autres dépenses hors du stade } \\
\text { (journaux, vêtements, souvenirs...) }\end{array}$ & & & \\
\hline q14i Dépense globale & & & \\
\hline
\end{tabular}

(enquêteur, jour, etc.), par une appréciation de la qualité des réponses perçue par l'enquêteur.

Sur la base des réponses données par les spectateurs, il a été possible de calculer la dépense moyenne individuelle pour une partie puis de passer à l'injection nette à l'aide de la formule suivante :

INJ netteSPECT $i=D M O Y i X$ (NBSPECTi-NBTRANSi-

NBOCCASi) X TXVAi

Où INJ nette SPECTi est l'injection nette des spectateurs dans la région $i, D M O Y i$ est la dépense moyenne individuelle pour une partie dans la région $i, N B S P E C T i$ le nombre de spectateurs des parties organisées dans la région, NBTRANSi le nombre de spectateurs ayant déplacé leur voyage dans le temps, NBOCCASi le nombre de spectateurs considérés comme occasionnels, c'est-à-dire venus dans la région pour un autre motif que la Coupe du monde de rugby, et TxVAi le taux de valeur ajoutée régionale.

Comme l'indique la formule précédente, il faut néanmoins déduire deux types de dépenses de l'injection nette obtenue : celle des visiteurs occasionnels présents sur le territoire pour un autre motif et celles des spectateurs qui ont déplacé leur visite pour la faire coïncider avec les dates de l'événement. Les visites occasionnelles ont pu être cernées à partir d'une question sur le motif principal de la venue, et les déplacements temporels devaient être renseignés dans une autre question. Dans les deux cas, ces dépenses ne peuvent pas être considérées comme des injections, à moins qu'il soit possible d'isoler le montant spécifiquement dépensé à l'occasion des parties de la Coupe du monde de rugby, ce qui est en pratique très difficile à réaliser (Preuss, 2006). La proportion de spectateurs occasionnels ou effectuant un transfert temporel a pu être estimée à $5 \%$ en Île-de-France, $6 \%$ en Pays de la Loire et Rhône-Alpes, 7 \% en Midi-Pyrénées, $9 \%$ en Aquitaine, Nord-Pas de Calais et Provence-Alpes-Côte d'Azur, 16 \% en Languedoc-Roussillon.

Conformément à la théorie de la base, l'injection nette émane uniquement des spectateurs français hors région et des supporteurs étrangers, la dépense des régionaux étant donnée pour comparaison uniquement. Les étrangers représentent $78 \%$ de l'injection pour $22 \%$ aux Français hors région étudiée.

\section{Principaux résultats}

Deux résultats originaux émergent de l'analyse : d'une part, globalement, ce sont les dépenses des touristes étrangers qui permettent de comprendre les différences régionales dans l'ampleur de l'impact total de la Coupe du monde de rugby. D'autre part, la nationalité des touristes est une variable explicative clé de l'ampleur des dépenses.

\section{Le poids global des spectateurs étrangers Domination de l'impact touristique}

La structure de l'impact sur l'ensemble des huit régions hôtes montre une très forte domination de l'impact touristique, par rapport à l'impact organisation. Les dépenses des visiteurs (spectateurs «stade» et "écran», accompagnateurs), engendrent 70,3\% de l'impact économique total de la Coupe du monde de rugby, et les dépenses d'organisation, 29,7\%. 


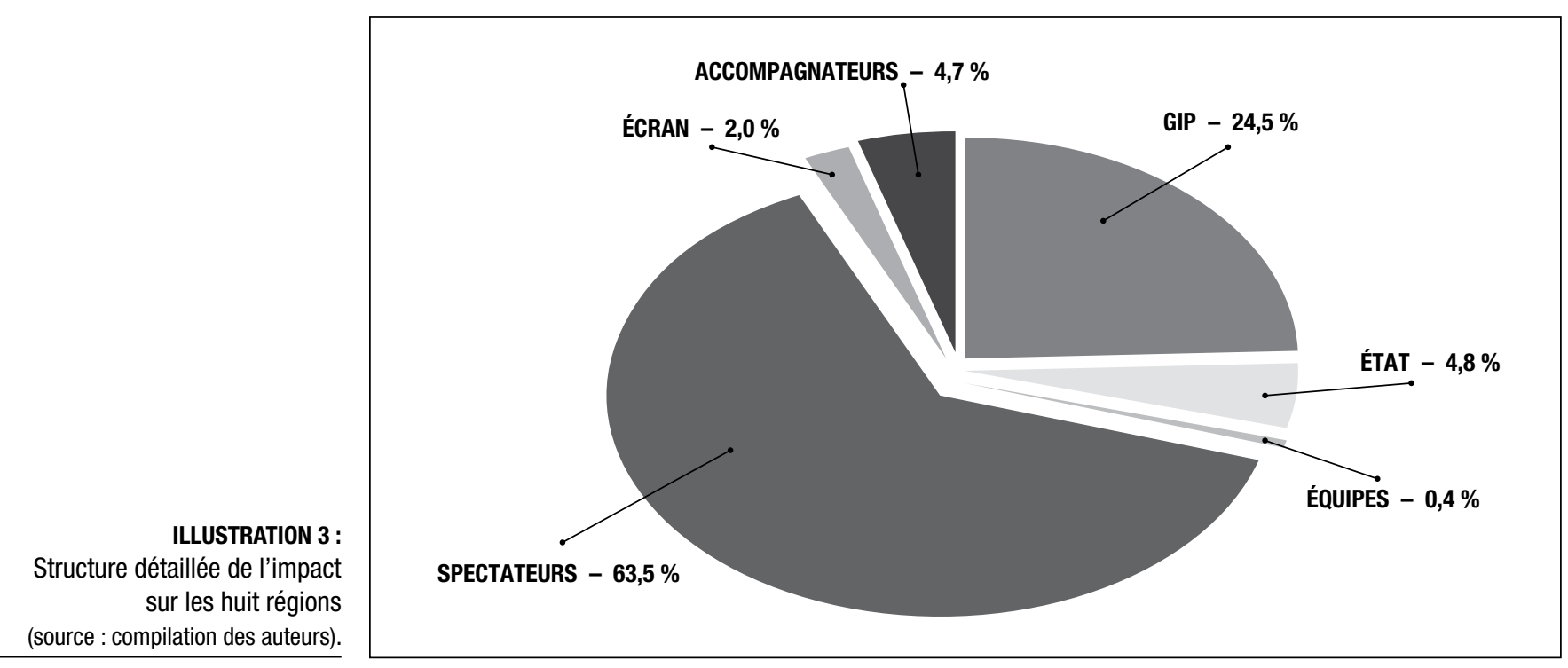

Tableau 1: Nature de l'impact touristique

\begin{tabular}{c|c|c|c|c}
\hline & $\begin{array}{c}\text { Impact } \\
\text { spectateurs } \\
\text { stade }\end{array}$ & $\begin{array}{c}\text { Impact } \\
\text { spectateurs } \\
\text { écrans }\end{array}$ & $\begin{array}{c}\text { Impact } \\
\text { accompagnateurs }\end{array}$ & $\begin{array}{c}\text { Impact } \\
\text { total } \\
\text { tourisme }\end{array}$ \\
\hline Aquitaine & $97,5 \%$ & $1,5 \%$ & $1,0 \%$ & $100,0 \%$ \\
\hline Île-de-France & $93,8 \%$ & $0,6 \%$ & $5,6 \%$ & $100,0 \%$ \\
\hline Languedoc-Roussillon & $88,5 \%$ & $2,4 \%$ & $9,1 \%$ & $100,0 \%$ \\
\hline Midi-Pyrénées & $68,5 \%$ & $22,1 \%$ & $9,4 \%$ & $100,0 \%$ \\
\hline Nord-Pas de Calais & $91,5 \%$ & $0,0 \%$ & $8,5 \%$ & $100,0 \%$ \\
\hline Pays de la Loire & $85,6 \%$ & $1,9 \%$ & $12,4 \%$ & $100,0 \%$ \\
\hline Provence-Alpes-Côte d'Azur & $88,6 \%$ & $4,8 \%$ & $6,6 \%$ & $100,0 \%$ \\
\hline Rhône-Alpes & $90,3 \%$ & $0,4 \%$ & $9,3 \%$ & $100,0 \%$ \\
\hline Source : compilation des auteurs.
\end{tabular}

On constate (voir illustration 3) que l'impact résulte d'ailleurs pour près des deux tiers $(63,5 \%)$ des dépenses des spectateurs qui sont entrés au stade tandis que les dépenses réalisées par le comité d'organisation génèrent un peu moins du quart $(24,5 \%)$ de l'impact global. La structure de l'impact de la Coupe du monde de rugby est donc aisée à analyser, avec une polarisation sur deux types de dépenses, et d'ailleurs principalement sur une seule. La faiblesse des retombées liées à l'implication de l'État s'explique par les investissements limités en infrastructures qui ont été réalisés. La Coupe du monde de rugby apparaît ainsi comme un événement à forte attractivité touristique, et très peu comme un outil d'aménagement pour les territoires d'accueil. Cette caractéristique le distingue nettement des Jeux olympiques et de la Coupe de monde de football qui sont l'occasion de gros travaux.

Le tableau 1 présenté ci-dessus détaille la structure interne de l'impact touristique, il montre une certaine homogénéité entre les régions, avec une forte domination des dépenses des spectateurs. En effet, de façon générale, le poids des dépenses des spectateurs "écrans géants » et des accompagnateurs est très faible. Cela signifie que la Coupe du monde de rugby en France a certes introduit l'innovation des écrans géants comme nouvelle forme de consommation des spectacles sportifs (885 800 supporteurs au total), mais que ce phénomène n'a pas encore atteint l'ampleur qu'il connaît dans d'autres sports et dans d'autres pays, comme le football en Grande Bretagne. On peut assister dans ce dernier pays à des déplacements de supporteurs qui, faute de places dans le stade ou de moyens financiers pour y pénétrer, viennent sur place suivre la compétition tout en bénéficiant de l'ambiance en direct, certes, mais également de la possibilité de consommer des produits interdits à l'intérieur de l'enceinte.

\section{Importance des spectateurs étrangers}

En poussant l'analyse plus loin, on constate que l'injection issue des spectateurs «stades» est principalement le fait des étrangers, comme le montre le tableau 2. 


\begin{tabular}{c|c|c}
\hline \multicolumn{3}{|c}{ Tableau 2: Part des étrangers dans l'injection spectateurs «stades " } \\
\hline Régions & $\begin{array}{c}\text { Part des étrangers } \\
\text { dans l'injection nette } \\
\text { spectateurs « stade " }\end{array}$ & $\begin{array}{c}\text { Part des étrangers } \\
\text { dans les spectateurs } \\
\text { extérieurs }\end{array}$ \\
\hline Aquitaine & $90,9 \%$ & $58,1 \%$ \\
\hline Île-de-France & $71,5 \%$ & $40,5 \%$ \\
\hline Languedoc-Roussillon & $80,0 \%$ & $52,6 \%$ \\
\hline Midi-Pyrénées & $71,1 \%$ & $26,8 \%$ \\
\hline Nord-Pas de Calais & $89,7 \%$ & $58,3 \%$ \\
\hline Pays de Loire & $89,3 \%$ & $57,4 \%$ \\
\hline Provence-Alpes-Côte d'Azur & $79,2 \%$ & $42,3 \%$ \\
\hline Rhône-Alpes & $65,9 \%$ & $44,9 \%$ \\
\hline
\end{tabular}

Source : compilation des auteurs.

En Aquitaine, si les étrangers représentent $58,1 \%$ des spectateurs hors région, ils ont été à l'origine de 90,9\% de l'injection nette spectateurs «stade». Cela s'explique par un panier moyen de dépenses des étrangers très supérieur à celui des Français. Un tel décalage dans toutes les régions entre masse de spectateurs et ampleur de dépenses atteint son maximum dans la région Midi-Pyrénées : les étrangers représentent seulement 26,8\% des spectateurs concernés mais leur injection pèse pour $71,1 \%$ du total. Le cas de cette région montre plus encore que l'ampleur de l'injection nette spectateurs «stades» provient très largement du poids des étrangers appartenant à la catégorie supérieure de revenu, soit plus de $4000 €$ de revenu moyen dans notre échantillon (voir tableau 3 ).

Avec un panier moyen de 553,63€ (comparé aux 44,95€ de la tranche inférieure de revenu des spectateurs français hors région), la tranche supérieure de revenu est à l'origine de $77,7 \%$ de l'injection nette des étrangers. Le revenu joue un rôle décisif dans le montant consommé comme le montrent les comparaisons que nous avons établies entre les spectateurs ayant plus ou moins de $4000 €$ de revenu mensuel pour le ménage. À titre d'illustration, en Provence-Alpes-Côte d'Azur, un étranger aisé dépense en moyenne $2021 €$ pour la famille, tandis que la consommation se réduit à $1147 €$ s'il dispose de moins de moyens. Des écarts du même type se retrouvent dans les autres régions hôtes (de $682 €$ à $433 €$ en Midi-Pyrénées, et de $906 €$ à $520 €$ en Languedoc-Roussillon). On mesure ici toute l'importance de l'attractivité de l'affiche ou de l'attrait du territoire vis-à-vis des étrangers à haut pouvoir d'achat.

\section{Deux types $d^{\prime}$ effets}

Le tableau 4 montre que l'ampleur de l'impact de la Coupe du monde de rugby dans chaque région dépend de deux variables:

- le nombre de billets vendus, que nous appelons effet taille.

- la répartition des billets entre locaux et étrangers, que nous appelons effet structure.

En reprenant le classement des régions par ordre décroissant d'impact, il est possible de synthétiser ces deux effets (voir tableau 5).

Le cas de l'Île-de-France, de loin première en termes d'impact économique, est très particulier, du fait du nombre de billets vendus sans commune mesure avec les autres régions, et également parce que l'impact des dépenses d'organisation a été pratiquement aussi important que celui des dépenses touristiques (ces dernières représentent $53 \%$ de l'impact total). Il ne faut pas oublier que l'organisation de la manifestation était particulièrement centralisée et que le comité d'organisation, le Groupement d'Intérêt Public France 2007, était situé à Paris. La répartition des spectateurs en fonction de leur provenance est proche de la moyenne des huit régions, ce n'est donc pas un effet structure qui est déterminant ici mais un effet taille (771 104 billets vendus).

L'impact enregistré par Provence-Alpes-Côte d'Azur (PACA) résulte des dépenses touristiques à 92,9\%. Cet impact s'explique à la fois par un effet taille (324 377 spectateurs) et un effet structure, la proportion de spectateurs locaux étant faible $(38,1 \%$ pour $47,9 \%$ sur l'ensemble des régions hôtes). La part des étrangers est très légèrement supérieure à la moyenne (et notamment les spectateurs hors Europe, les ressortissants du Pacifique représentant plus du quart des étrangers).

L'impact sur Rhône-Alpes peut apparaître décevant au regard du nombre de billets disponibles (230 300 spectateurs) sur deux sites (Saint-Étienne et Lyon). Cela s'explique essentiellement par une forte proportion de spectateurs locaux $(55,3 \%)$. Néanmoins, la part inférieure à la moyenne des huit régions en ce qui concerne les spectateurs étrangers a été compensée par le fait qu'il s'agit pour plus de la moitié de non-Européens (ayant une dépense plus élevée).

L'Aquitaine bénéficie de retombées pratiquement au niveau de celles de Rhône-Alpes malgré un nombre de billets (133 292) près de deux fois inférieur (effet taille négatif). Cela résulte d'une plus forte représentation des spectateurs étrangers dans le public. Les mêmes remarques peuvent être faites pour Nord-Pas de Calais et Languedoc-Roussillon.

Les impacts les plus faibles sont enregistrés par les Pays de la Loire et Midi-Pyrénées, régions qui ont cumulé un effet taille négatif (109 156 billets pour la première, et 138690 pour la seconde), et un effet structure défavorable. Pour Pays de Loire, la quasi non-représentation des spectateurs non européens explique la faiblesse de l'impact. En Midi-Pyrénées, la très forte présence de spectateurs locaux ne créant pas de valeur ajoutée $(59,4 \%)$, associée à une très faible proportion de visiteurs étrangers (10,9\%), explique que l'activité économique n'ait pas été affectée à hauteur des espérances.

\section{La nationalité, variable explicative clé Analyse des paniers de dépenses}

La nationalité constitue une variable explicative clé du montant et de la structure des dépenses des spectateurs (voir tableau 6). Nous sommes parvenus à ce constat sans prendre en compte les différentiels de taux de change. Or, on sait que, pour effectuer des comparaisons internationales, on peut utiliser un mode de calcul basé sur la parité de pouvoir d'achat (PPA). Il faut pour cela établir un panier de biens et services normalisé qui peut poser problème. En s'affranchissant des taux de change on fait reposer la validité de la comparaison sur la qualité de la définition du panier. On mesure ici toute l'ampleur de la difficulté des comparaisons internationales en matière de niveau de vie. Pour cette raison, nous en sommes 
Tableau 3: Injection nette par catégorie de spectateurs en Midi-Pyrénées

\begin{tabular}{|c|c|c|c|c|c|}
\hline $\begin{array}{l}\text { Spectateurs } \\
\text { hors région }\end{array}$ & $\begin{array}{l}\text { Nombre de } \\
\text { spectateurs }\end{array}$ & $\begin{array}{c}\% \text { des } \\
\text { spectateurs }\end{array}$ & $\begin{array}{c}\text { Panier de } \\
\text { dépenses }(\xi)\end{array}$ & $\begin{array}{c}\text { Montant } \\
\text { injection }(€)\end{array}$ & $\begin{array}{c}\% \text { de } \\
\text { l'injection }\end{array}$ \\
\hline $\begin{array}{c}\text { Revenu inférieur à } \\
4000 € \text { / mois }\end{array}$ & 27257 & 66,1 & 44,9 & 738014 & 51,3 \\
\hline $\begin{array}{l}\text { Revenu supérieur à } \\
4000 € / \text { mois }\end{array}$ & 13968 & 33,9 & 83,1 & 699145 & 48,7 \\
\hline Total & 41225 & 100,0 & & 1437159 & 100,0 \\
\hline Étrangers & $\begin{array}{l}\text { Nombre de } \\
\text { spectateurs }\end{array}$ & $\begin{array}{c}\text { \% des } \\
\text { spectateurs }\end{array}$ & $\begin{array}{c}\text { Panier de } \\
\text { dépenses }(\xi)\end{array}$ & $\begin{array}{c}\text { Montant } \\
\text { injection }(€)\end{array}$ & $\begin{array}{c}\% \text { de } \\
\text { l'injection }\end{array}$ \\
\hline $\begin{array}{l}\text { Revenu inférieur à } \\
4000 € / \text { mois }\end{array}$ & 7030 & 46,3 & 194,7 & 824392 & 22,3 \\
\hline $\begin{array}{c}\text { Revenu supérieur à } \\
4000 € / \text { mois }\end{array}$ & 8121 & 53,7 & 553,6 & 2708233 & 77,7 \\
\hline Ensemble étrangers & 15151 & 100,0 & & 3532625 & 100,0 \\
\hline
\end{tabular}

Tableau 4: Les facteurs explicatifs des disparités régionales d'impact

\begin{tabular}{c|c|c|c|c|c|c|c|c}
\hline $\begin{array}{c}\text { Régions } \\
\text { d'accueil }\end{array}$ & $\begin{array}{c}\text { Impact } \\
\text { total }\end{array}$ & $\begin{array}{c}\text { Impact } \\
\text { tourisme }\end{array}$ & $\begin{array}{c}\% \text { impact } \\
\text { tourisme }\end{array}$ & $\begin{array}{c}\text { Nombre de } \\
\text { rencontres }\end{array}$ & $\begin{array}{c}\text { Nombre } \\
\text { billets }\end{array}$ & $\begin{array}{c}\% \text { billets } \\
\text { locaux }\end{array}$ & $\begin{array}{c}\% \text { billets } \\
\text { étrangers }\end{array}$ & $\begin{array}{c}\% \text { billets } \\
\text { tri nations }\end{array}$ \\
\hline Aquitaine & 38953987 & 32391662 & $83,2 \%$ & 4 & 133292 & $51,2 \%$ & $28,4 \%$ & $28,01 \%$ \\
\hline Île-de-France & 253791824 & 135490664 & $53,4 \%$ & 12 & 771104 & $46,0 \%$ & $21,9 \%$ & $16,54 \%$ \\
\hline Languedoc-Roussillon & 30840970 & 23035417 & $74,7 \%$ & 4 & 111607 & $46,6 \%$ & $28,1 \%$ & $52,77 \%$ \\
\hline Midi-Pyrénées & 20733089 & 14659424 & $70,7 \%$ & 4 & 138690 & $59,4 \%$ & $10,9 \%$ & $42,65 \%$ \\
\hline Nord-Pas de Calais & 32157761 & 24456850 & $76,1 \%$ & 3 & 107786 & $56,3 \%$ & $35,7 \%$ & $9,63 \%$ \\
\hline Pays de la Loire & 27387742 & 21792820 & $79,6 \%$ & 3 & 109156 & $45,3 \%$ & $31,4 \%$ & $1,76 \%$ \\
\hline Provence-Alpes-Côte d'Azur & 145350165 & 135083035 & $92,9 \%$ & 6 & 324377 & $38,1 \%$ & $26,2 \%$ & $19,55 \%$ \\
\hline Rhône-Alpes & 40506456 & 27517425 & $67,9 \%$ & 6 & 230300 & $55,3 \%$ & $20,1 \%$ & $39,15 \%$ \\
\hline AGRÉGATION & 589721995 & 414427298 & $70,3 \%$ & 42 & 1926312 & $47,9 \%$ & $23,4 \%$ & $23,30 \%$ \\
\hline
\end{tabular}

Source : compilation des auteurs.

restés à l'analyse brute des paniers de dépenses, étant entendu que l'ampleur et la nature de telles dépenses est plus conditionnée, dans notre cas bien spécifique de supporteurs de rugby, par leur culture que par leur pouvoir d'achat.

Les dépenses les plus élevées sont le fait des spectateurs originaires du Pacifique avec l'Australie (4 $488 €$ en moyenne), ou la Nouvelle-Zélande et les autres îles pacifiques (4 $127 €$ ). Les ressortissants des autres continents lointains ont également des dépenses d'un montant élevé : l'Asie et l'Amérique du sud (Argentins) avec $3341 €$; l'Afrique avec $2596 €$ (essentiellement l'Afrique de Sud); l'Amérique du Nord avec $2301 €$. Il apparaît ainsi que les dépenses des Européens sont significativement plus faibles. Ce sont les ressortissants britanniques qui dépensent le plus avec $1274 €$, ce qui représente quasiment le double du niveau des dépenses des autres Européens avec $644 €$. Dans ce contexte, la France fait figure de faible dépensière avec $401 €$ pour les Français hors région d'accueil et seulement $75 €$ pour les spectateurs locaux.

\begin{tabular}{c|c|c}
\hline \multicolumn{3}{c}{$\begin{array}{c}\text { Tableau 5: Synthèse des éléments déterminants } \\
\text { de l'ampleur de l'impact économique }\end{array}$} \\
\hline Régions d'accueil & Effet taille & Effet structure \\
\hline île-de-France & +++ & $=$ \\
\hline Provence-Alpes-Côte d'Azur & ++ & ++ \\
\hline Rhône-Alpes & + & - \\
\hline Aquitaine & - & + \\
\hline Nord-Pas de Calais & - & + \\
\hline Languedoc-Roussillon & - & + \\
\hline Pays de Loire & - & $=$ \\
\hline Midi-Pyrénées & - & -- \\
\hline
\end{tabular}

Source : compilation des auteurs. 
Tableau 6: Montant et structure de dépenses par nationalité

\begin{tabular}{|c|c|c|c|c|c|c|c|c|c|c|}
\hline \multirow{2}{*}{ Groupe pays } & \multirow{2}{*}{$\begin{array}{c}\text { Dépense } \\
\text { moyenne ( } € \text { ) }\end{array}$} & \multirow{2}{*}{$\begin{array}{c}\% \\
\text { transport }\end{array}$} & \multirow{2}{*}{$\begin{array}{l}\text { Dépense hors } \\
\text { transport }\end{array}$} & \multicolumn{7}{|c|}{$\%$ de la dépense hors transport constituée par... } \\
\hline & & & & Logement & Achats stade & Nourriture & Sorties & Tourisme & Services & Autres \\
\hline Afrique & 2596 & 33,4 & 1728 & 42,3 & 7,0 & 26,9 & 12,9 & 3,4 & 0,4 & 7,1 \\
\hline Amérique du Nord & 2301 & 34,3 & 1511 & 36,3 & 11,6 & 23,8 & 9,1 & 6,4 & 1,1 & 11,6 \\
\hline Australie & 4488 & 30,3 & 3127 & 39,2 & 7,0 & 27,2 & 13,0 & 4,6 & 1,0 & 8,0 \\
\hline Autre (AS, A) & 3341 & 40,6 & 1986 & 36,2 & 10,6 & 19,5 & 11,3 & 11,4 & 0,5 & 10,6 \\
\hline Îles Britanniques & 1274 & 33,7 & 845 & 36,0 & 11,1 & 24,7 & 17,1 & 3,4 & 0,6 & 7,0 \\
\hline Reste de l'Europe & 644 & 29,8 & 452 & 24,5 & 11,2 & 31,1 & 15,3 & 4,3 & 0,6 & 13,0 \\
\hline Français hors région & 401 & 40,9 & 237 & 12,5 & 17,3 & 26,8 & 8,9 & 27,3 & 0,4 & 6,7 \\
\hline Français région & 75 & 42,4 & 43 & 2,4 & 45,0 & 27,7 & 8,0 & 9,4 & 0,2 & 7,4 \\
\hline Pacifique & 4127 & 41,8 & 2402 & 48,7 & 10,7 & 18,3 & 9,8 & 3,5 & 0,9 & 8,0 \\
\hline Total général & 633 & 40,0 & 380 & 15,1 & 27,3 & 26,8 & 10,3 & 12,7 & 0,4 & 7,5 \\
\hline
\end{tabular}

Source : compilation des auteurs.

\begin{tabular}{|c|c|c|c|}
\hline Groupe pays & $\begin{array}{l}\text { Dépense moyenne } \\
\text { hors transport }(€)\end{array}$ & $\begin{array}{l}\text { Nombre moyen } \\
\text { de parties vues }\end{array}$ & $\begin{array}{l}\text { Dépense moyenne } \\
\text { par partie }\end{array}$ \\
\hline Afrique & 1728,4 & 2,7 & 643 \\
\hline Amérique du Nord & 1510,6 & 2,2 & 690 \\
\hline Australie & 3127,4 & 3,1 & 1009 \\
\hline Autre (AS, A) & 1986,3 & 2,4 & 834 \\
\hline Îles Britanniques & 844,7 & 2,5 & 339 \\
\hline Reste de l'Europe & 452,3 & 1,8 & 257 \\
\hline Français hors région & 237,1 & 2,3 & 103 \\
\hline Français région & 42,9 & 2,2 & 19 \\
\hline Pacifique & 2402,5 & 2,9 & 834 \\
\hline Total général & 379,9 & 2,3 & 165 \\
\hline \multicolumn{4}{|c|}{ Source : compilation des auteurs. } \\
\hline
\end{tabular}

Au-delà de la dépense globale, quand on analyse le panier de dépenses par partie et hors dépense de transport, les écarts de nationalités subsistent mais avec quelques nuances (voir tableau 7). Les Australiens sont de loin les plus dépensiers parmi les ressortissants des pays les plus lointains. Les spectateurs britanniques dépensent le tiers d'un Australien et $25 \%$ de plus environ qu'un spectateur du reste de l'Europe. La dépense moyenne des Français, quant à elle, pourrait permettre de s'interroger sur le niveau de la culture sportive dans notre pays.

Si le montant absolu de la dépense donne des éléments intéressants de comparaison entre les nationalités, il faut néanmoins noter une relative homogénéité, à quelques exceptions près, de la structure de la dépense totale des spectateurs par grands postes, quelle que soit la nationalité. Les dépenses de transport pèsent entre 30 et $42 \%$ de la dépense totale, et les dépenses hors transport se répartissent entre le logement, la nourriture, les sorties avec quelques spécificités nationales : a) les Français résidant dans les régions d'accueil ont un niveau de dépenses au stade très supérieur à la moyenne générale $(45 \%$ contre $27,3 \%)$. Il ne faut pas oublier néanmoins les montants sur lesquels portent ces pourcentages. La faiblesse de la dépense individuelle française $(43 €)$ fait qu'un Australien, avec seulement $7 \%$ de sa dépense totale, injecte dix fois plus d'argent dans le stade qu'un local;

b) les Français hors région consacrent plus des trois quarts de leur budget hors transport au tourisme (27,3\%), ce qui les singularise du reste des spectateurs (avec une moyenne de $12,7 \%$ ) ;

c) pour les sorties, les supporteurs des îles Britanniques et du reste de l'Europe s'écartent notablement de la moyenne nationale de $10,3 \%$ avec respectivement $17,1 \%$ et $15,3 \%$;

d) les ressortissants des pays les plus lointains se distinguent par des dépenses de logement très supérieures à la moyenne $(15,1 \%)$, ce qui est logique. Le maximum est atteint pour les ressortissants des îles pacifiques qui consacrent près de la moitié de leur budget à l'hébergement.

On constate donc que les montants dépensés sont fortement hétérogènes selon les nationalités. Dans la mesure où les supporteurs étrangers se concentrent dans les villes dans laquelle va évoluer leur équipe nationale, comme en témoigne le tableau 8 , les régions gagnantes sont notamment celles qui ont la chance de s'être vu attribuer des parties impliquant des équipes de l'hémisphère sud (Île-de-France et Rhône-Alpes en tête). En termes de prospective, ces résultats signifient que la nationalité des spectateurs pourrait constituer une variable d'ajustement importante dans la répartition régionale de l'impact de la Coupe du monde de rugby puisqu'il suffirait d'attirer les nationalités les plus dépensières. Néanmoins, la programmation sportive est exogène aux régions d'accueil et obéit à d'autres critères. Il serait intéressant de discuter au niveau de l'organisation générale des grands événements sportifs si des éléments autres que sportifs peuvent rentrer dans la grille d'attribution des parties. 
Tableau 8: Répartition des spectateurs par nationalité et par site de compétition

\begin{tabular}{|c|c|c|c|c|c|c|c|c|c|c|c|c|c|}
\hline \multirow{2}{*}{$\begin{array}{c}\text { Somme ventes } \\
\text { Pays }\end{array}$} & \multicolumn{13}{|c|}{ Lieu de compétition } \\
\hline & Bordeaux & Lens & Lyon & Marseille & Montpellier & Nantes & Paris & $\begin{array}{l}\text { Saint- } \\
\text { Denis }\end{array}$ & $\begin{array}{c}\text { Saint- } \\
\text { Étienne }\end{array}$ & Toulouse & Total 1 & Voyagistes & Total 2 \\
\hline Royaume-Uni & 8075 & 17690 & 5226 & 28299 & 6444 & 24683 & 33500 & 43488 & 4920 & 4175 & 176500 & 41567 & 218067 \\
\hline Australie & 2583 & 144 & 2316 & 3148 & 3125 & 104 & 474 & 1549 & 485 & 337 & 14265 & 39727 & 53992 \\
\hline Nouvelle-Zélande & 40 & 24 & 702 & 1258 & 132 & 7 & 97 & 1286 & 66 & 659 & 4271 & 26496 & 30767 \\
\hline Irlande & 8702 & 186 & 235 & 913 & 349 & 184 & 7143 & 5403 & 125 & 227 & 23467 & 5063 & 28530 \\
\hline Afrique du Sud & 3 & 350 & 6 & 556 & 424 & 6 & 489 & 802 & 10 & 10 & 2656 & 14378 & 17034 \\
\hline États-Unis & 649 & 381 & 381 & 1183 & 967 & 164 & 918 & 1502 & 467 & 150 & 6762 & 9490 & 16252 \\
\hline Italie & 57 & 33 & 229 & 6944 & 153 & 22 & 386 & 571 & 3142 & 46 & 11583 & & 11583 \\
\hline Espagne & 586 & 95 & 340 & 2306 & 898 & 150 & 371 & 931 & 346 & 519 & 6542 & & 6542 \\
\hline Belgique & 116 & 2527 & 170 & 530 & 211 & 34 & 916 & 1223 & 105 & 66 & 5898 & & 5898 \\
\hline Suisse & 159 & 79 & 1371 & 940 & 274 & 39 & 412 & 1074 & 602 & 121 & 5071 & & 5071 \\
\hline Allemagne & 186 & 505 & 483 & 928 & 428 & 79 & 515 & 711 & 226 & 214 & 4275 & & 4275 \\
\hline Japon & 192 & 38 & 189 & 119 & 47 & 4 & 154 & 273 & 19 & 156 & 1191 & 2997 & 4188 \\
\hline Argentine & 3 & & 125 & 418 & 29 & 2 & 164 & 611 & 9 & 1 & 1362 & 2328 & 3690 \\
\hline Pays-Bas & 140 & 940 & 89 & 528 & 168 & 61 & 477 & 633 & 146 & 87 & 3269 & & 3269 \\
\hline Canada & 622 & 80 & 90 & 363 & 134 & 56 & 179 & 352 & 76 & 45 & 1997 & 719 & 2716 \\
\hline Hong-Kong & 46 & 27 & 26 & 133 & 48 & 8 & 85 & 161 & 43 & 28 & 605 & 1200 & 1805 \\
\hline Portugal & 35 & 10 & 359 & 149 & 112 & 2 & 141 & 85 & 280 & 222 & 1395 & & 1395 \\
\hline Luxembourg & 78 & 60 & 70 & 178 & 51 & 21 & 230 & 412 & 54 & 33 & 1187 & & 1187 \\
\hline Autres & 352 & 215 & 621 & 1635 & 435 & 178 & 615 & 1842 & 240 & 378 & 6511 & 0 & 6511 \\
\hline Total étrangers par site & 22624 & 23384 & 13028 & 50528 & 14429 & 25804 & 47266 & 62909 & 11361 & 7474 & 278807 & 143965 & 422772 \\
\hline
\end{tabular}

\section{Analyse des profils sociodémographiques}

Pour comprendre les résultats obtenus sur les injections des spectateurs étrangers et sur leurs paniers de dépenses, il est nécessaire de connaître leur profil sociodémographique. En effet, il apparaît que les comportements de dépense des différentes nationalités varient considérablement en fonction de variables caractérisant des valeurs culturelles. En conséquence, ce n'est pas tant l'origine géographique des spectateurs qui compte mais leur culture nationale ou continentale. Pour ce faire, nous regrouperons les spectateurs étrangers en trois catégories classées par ordre décroissant du montant de leurs dépenses : les ressortissants extra-européens; les Européens; les Français.

\section{Ressortissants extra-européens}

En termes de paniers de dépenses, le classement pour les ressortissants extra-européens est le suivant : 1) Australiens; 2) Néo-Zélandais et îles pacifiques; 3) Asiatiques et SudAméricains («autres»); 4) Africains; 5) Nord-Américains. Tous ces spectateurs ont déclaré un revenu moyen supérieur à $4500 €$. Des différenciations apparaissent ensuite au niveau des autres variables, comme le montre le tableau de synthèse suivant (voir tableau 9).

Si l'on compare tout d'abord les supporteurs en provenance du Pacifique, il apparaît qu'il y a moins d'Australiens possédant un diplôme d'études supérieures $(30,8 \%)$ que de Néo-Zélandais et de ressortissants des îles pacifiques (45,5\%), ces pourcentages restant inférieurs à la moyenne de l'ensemble du public $(47,7 \%)$. Ces deux populations sont plus âgées que la moyenne (43 ans contre 39) et ce sont des partisans de rugby ou des sportifs en général. Les Australiens assistent à huit parties de rugby par an (contre 4,8 en moyenne générale); un tiers des Néo-Zélandais pratiquent le rugby et ils assistent à 11,2 parties de rugby par an.

Les Asiatiques et Sud-Américains se distinguent principalement par un niveau d'études supérieures plus élevé $(57,1 \%)$, un pourcentage d'hommes légèrement plus fort que la moyenne et un pourcentage de pratiquants de rugby plus élevé. Les Sud-Africains sont relativement moins diplômés d'études supérieures mais sont surreprésentés par rapport à la moyenne au niveau de l'assistance à des spectacles sportifs ainsi que de la pratique sportive.

De la même façon, les Nord-Américains ont un profil marqué de pratiquants sportifs mais d'un pourcentage de diplômés d'études supérieures le plus faible de l'ensemble ( $16,7 \%$ contre $47,7 \%$ en moyenne).

\section{Ressortissants européens}

Deux caractéristiques prédominent pour les ressortissants européens : les spectateurs des îles Britanniques (les plus nombreux) disposent de revenus élevés mais d'un taux de diplômés d'études supérieures en dessous de la moyenne de l'échantillon (32,1\% contre 47,7\%). Les spectateurs des autres pays européens se distinguent des Britanniques par un revenu nettement plus faible ( $3195 €$ contre 4 805), ils sont plus jeunes (36 ans en moyenne, soit les plus jeunes, toutes nationalités confondues) et leur taux de pratique du rugby est plus fort avec $29 \%$. 
Tableau 9: Le profil socio-économique des spectateurs de la Coupe du monde de rugby

\begin{tabular}{|c|c|c|c|c|c|c|c|c|c|c|}
\hline Groupe pays & Afrique & $\begin{array}{c}\text { Amérique } \\
\text { du Nord }\end{array}$ & Australie & Autre $(\mathrm{AS}, \mathrm{A})$ & $\begin{array}{c}\text { Îles } \\
\text { Britanniques }\end{array}$ & $\begin{array}{l}\text { Reste de } \\
\text { l'Europe }\end{array}$ & $\begin{array}{l}\text { Français } \\
\text { hors région }\end{array}$ & $\begin{array}{c}\text { Français } \\
\text { région }\end{array}$ & Pacifique & Ensemble \\
\hline \multicolumn{11}{|l|}{ PROFIL } \\
\hline$\%$ hommes & 76,6 & 74,5 & 74,6 & 82,0 & 77,3 & 78,3 & 80,0 & 78,7 & 73,3 & 78,6 \\
\hline$\%$ mariés ou concubins & 70,1 & 63,6 & 68,1 & 60,0 & 62,4 & 62,6 & 70,4 & 67,8 & 68,2 & 67,7 \\
\hline$\%$ études supérieures & 25,7 & 16,7 & 30,8 & 57,1 & 32,1 & 35,5 & 53,6 & 50,7 & 45,5 & 47,7 \\
\hline Revenu moyen & 5232 & 4943 & 5369 & 4506 & 4805 & 3195 & 2027 & 1825 & 4622 & 2455 \\
\hline Âge moyen & 39 & 38 & 43 & 36 & 40 & 36 & 39 & 39 & 43 & 39 \\
\hline \multicolumn{11}{|l|}{ PROFIL SPORTIF } \\
\hline Nombre de spectacles sportifs & 12,3 & 11,6 & 11,7 & 12,1 & 9,2 & 8,8 & 7,5 & 7,8 & 14,3 & 8,5 \\
\hline Nombre de spectacles de rugby & 8,6 & 5,0 & 8,0 & 4,6 & 6,2 & 5,2 & 4,6 & 3,8 & 11,2 & 4,8 \\
\hline$\%$ de pratiquants de rugby & 18,1 & 35,2 & 20,7 & 36,2 & 21,0 & 29,7 & 19,8 & 16,4 & 32,6 & 19,2 \\
\hline$\%$ pratiquants sportifs & 72,4 & 81,1 & 64,5 & 55,1 & 65,0 & 61,8 & 61,7 & 63,9 & 64,4 & 63,6 \\
\hline \multicolumn{11}{|l|}{ TYPE DE GROUPE } \\
\hline$\%$ venu en famille & 51,4 & 62,0 & 56,3 & 48,8 & 49,1 & 42,9 & 60,7 & 55,8 & 65,0 & 55,9 \\
\hline Nombre moyen membres famille & 1,2 & 1,6 & 1,3 & 1,2 & 1,1 & 1,5 & 1,2 & 1,3 & 1,5 & 1,3 \\
\hline Nombre moyen accompagnateurs & 7,2 & 7,3 & 7,8 & 5,9 & 3,9 & 3,4 & 3,6 & 3,6 & 5,0 & 3,9 \\
\hline Nombre moyen de jeunes & 1,5 & 0,4 & 0,9 & 0,3 & 0,2 & 0,3 & 0,3 & 0,9 & 0,3 & 0,6 \\
\hline \multicolumn{11}{|l|}{ HÉBERGEMENT } \\
\hline Nombre moyen de nuits & 9,2 & 11,9 & 17,8 & 5,2 & 7,2 & 4,0 & 0,7 & - & 16,1 & 4,0 \\
\hline \% hébergé en hôtel & 86,0 & 71,1 & 83,9 & 82,9 & 80,3 & 63,4 & 50,4 & - & 78,9 & 67,1 \\
\hline Dépense moyenne logement $(€)$ & 875,3 & 818,8 & 1402,0 & 935,6 & 436,5 & 179,6 & 109,2 & - & 1360,0 & 235,7 \\
\hline \multicolumn{11}{|l|}{ TOURISME } \\
\hline $1^{\text {re }}$ visite en France & 48,9 & 56,3 & 45,5 & 48,7 & 13,5 & 16,2 & - & - & 48,4 & 27,9 \\
\hline$\%$ pensant revenir & 80,0 & 53,3 & 60,0 & 63,2 & 94,0 & 97,3 & - & - & 83,9 & 85,0 \\
\hline
\end{tabular}

Ressortissants français

Les deux catégories de spectateurs français (résidant ou non dans la région d'accueil) ont des profils relativement proches. Ils se distinguent fondamentalement des autres nationalités par deux caractéristiques : une proportion beaucoup plus forte de diplômés d'études supérieures et des revenus beaucoup plus faibles. Dans la mesure où l'événement se déroule en France, il y a un effet d'aubaine, l'événement étant accessible à des personnes n'ayant pas nécessairement de revenus très élevés bien que fortement diplômées. Par ailleurs, la culture sportive de ces spectateurs est peu éloignée de la moyenne.

En résumé, les variables qui apparaissent les plus discriminantes semblent être le revenu, le diplôme et la culture sportive. Il faut néanmoins combiner ce profil avec le nombre de spectateurs étrangers pour comprendre l'ampleur de l'impact régional de la Coupe du monde de rugby.

\section{Conclusion}

Les résultats obtenus montrent que la Coupe du monde de rugby a eu un impact économique positif dans toutes les régions, mais avec des écarts considérables tenant principalement à la présence de spectateurs étrangers « riches». Cette part relative forte de l'injection des touristes étrangers tient également au fait que la Coupe du monde de rugby n'a pas demandé de constructions nouvelles d'infrastructures sportives. L'impact de la Coupe du monde de rugby en région a donc été largement conditionné à la fois par l'affiche des compétitions et par l'attractivité générale du territoire pour des étrangers (Île-de-France ou Provence-Alpes-Côte d'Azur, par exemple).

Au final, les disparités régionales d'impact s'expliquent à la fois par un effet taille (nombre de billets vendus) et par un effet structure (parts relatives des différentes nationalités). Selon les régions, ces deux effets se confortent plus ou moins ou s'opposent. Le cas idéal est bien sûr la région qui reçoit beaucoup de rencontres et dont l'affiche attire beaucoup d'étrangers à hauts revenus et niveau de dépenses élevé. À l'inverse, certaines régions qui ont été faiblement pourvues en parties intéressantes ont pu compenser une partie de ce handicap grâce à une venue d'étrangers aisés attirés par leur équipe nationale.

L'impact économique régional de la Coupe du monde de rugby était donc très lié à la qualité de l'affiche d'une part et aux nationalités concernées d'autre part. Cela signifie qu'il pourrait être possible de penser une véritable politique d'accueil de ce type d'événement permettant de répartir au mieux l'impact économique total entre les différentes régions. Deux variables peuvent être modulées :

- le nombre de billets (ou de parties) attribués à chaque région;

- et le type de partie attribué quant à sa place dans le calendrier (éliminatoires ou phases finales) mais également quant aux nationalités concernées.

On comprend ainsi que la totalité de l'impact régional ne pourra pas être complètement maîtrisé. Si une partie des affiches peut être attribuée au début de la compétition par 
l'organisateur, une autre partie dépend ensuite des résultats sportifs et ne peut être programmée. À partir de là, les régions candidates peuvent faire des calculs d'anticipation pour évaluer les chances d'optimiser les retombées économiques sur leur territoire.

\section{Références}

BAADE, Robert (1996) «Professional sports as a catalyst for metropolitan economic development", Journal of urban affairs, vol. 18, $\mathrm{n}^{\circ}$ 1, p. 1-17.

BARGET, Eric et Jean-Jacques GOUGUET (2010a) Evénements sportifs : impact économique et social, coll. «Management et sport», Bruxelles : De Boeck. $462 \mathrm{p}$.

BARGET, Eric et Jean-Jacques GOUGUET (2010b) De l'évaluation des grands événements sportifs. La coupe du monde de rugby 2007 en France, Limoges : PULIM. 386 p.

BARGET, Eric et Jean-Jacques GOUGUET (2010c) «La mesure de l'impact économique des grands événements sportifs : l'exemple de la Coupe du monde de rugby 2007 ", Revue d'Économie Régionale et Urbaine, $\mathrm{n}^{\circ} 2$, p. 379-408.

BARGET, Eric et Jean-Jacques GOUGUET (2010d) «Une analyse coûtsbénéfices de la Coupe du Monde de Rugby 2007 en France : Une perspective régionale», Économie appliquée, vol. 63, n 3, p. 205-228.

BLAKE, Adam (2005) «The economic impact of the London 2012 Olympics», Christel DeHann Tourism and Travel Research Institute. Non publié. Nottingham : Nottingham University Business School.

BOURG, Jean-François et Jean-Jacques GOUGUET (1998) Analyse économique du sport, Paris : PUF. 380 p.

CHAPPELET, Jean-Loup (dir.) (2006) Les politiques publiques d'accueil d'événements sportifs, Paris : L'Harmattan, coll. «Questions contemporaines». $237 \mathrm{p}$.

COPELAND, B. R. (1991) «Tourism, Welfare and de-industrialisation in a small open economy", Economica, vol. 58, p. 515-529.

DAVIDSON, Larry S. (1999) «Choice of a proper methodology to measure quantitative and qualitative effects of the impact of sport», DANS The Economic Impact of Sport Events, sous la direction de JEANRENAUD, Claude, p. 28-44. Neuchâtel : Editions CIES.

DAVEZIES Laurent (2008) La république et ses territoires. La circulation invisible des richesses, Paris : Seuil. $112 \mathrm{p}$.

DION, Yves (1987) «Le multiplicateur régional appliqué à un espace économique de petite dimension ». Thèse de doctorat, Bordeaux : Université de Bordeaux I. 264 p.

DOUCET, Carole (2002) «Activités viticoles et développement régional». Thèse de doctorat en science économique, Bordeaux : Université de Montesquieu Bordeaux IV. 352 p.

DWYER, Larry; Peter FORSYTH et Ray SPURR (2003) «Inter-industry effects of tourism growth: implications for destination managers ", Tourism Economics, vol. 9, n² 2, p. 117-132.

ESSEC - Chaire européenne de marketing sportif (2007) Les retombées économiques de la Coupe du monde de rugby 2007 en France, étude pour le Comité d'organisation France 2007, Paris, 27 avril 2007.

GOUGUET, Jean-Jacques (1981) «Pour une réhabilitation de la théorie de la base», Revue d'économie régionale et urbaine, no 1, p. 63-83.
JEANRENAUD, Claude (dir.) (1999) The Economic Impact of Sport Events, Neuchâtel : Editions CIES. 210 p.

KASIMATI, Evangelia (2003) «Economic aspects of the Summer Olympics: a review of related research ", International Journal of Tourism Research, vol. $5, \mathrm{n}^{\circ} 6, \mathrm{p} .433-444$.

KESENNE, Stephan (2005) «Do we need an economic impact study or a cost-benefit analysis of sports event?», European Sport Management Quarterly, vol. 5, n² 2, p. 133-142.

LI, ShiNa et Adam BLAKE (2009) «Estimating Olympic Related Investments and Expenditure», International Journal of Tourism Research, vol. 11, $\mathrm{n}^{\mathrm{o}}$ 4, p. 337-356.

MADDEN, John Robert (2002) «The Economic Consequencies of the Sydney Olympics», The CREA/Arthur Andersen Study, Current Issues in Tourism, vol. 5, $\mathrm{n}^{\circ}$ 1, p. 7-21.

MANKIW, Gregory (1998) Principes de l'économie, Paris : Economica. 972 p.

MARTIN, Fernand (1991) «Faiblesses, embûches et abus dans les analyses avantages-coûts de projets", Revue canadienne d'études du développement, vol. 12, $\mathrm{n}^{\circ}$ 1, p. 90-105.

MKG HOSPITALITY (2007) Bilan de la Coupe du Monde de Rugby : l'hôtellerie française sort gagnante, communiqué de presse, 24 octobre, Paris.

PERRET Bernard (2004) Évaluation des politiques publiques, Paris : La Découverte. $126 \mathrm{p}$.

PORTER, Philip et Deborah FLETCHER (2008) «The Economic Impact of the Olympic Games: Ex-ante Predictions and Ex-poste Reality », Journal of Sport Management, vol. 22, $\mathrm{n}^{\circ}$ 4, p. 470-486.

PREUSS, Holger (dir.) (2006) «Special Issue : Impact and Evaluation of Major Sporting Events», European Sport Management Quarterly, vol. 6, $\mathrm{n}^{\circ} 4$, p. 313-415.

PREUSS, Holger (2007a) «The Conceptualisation and Measurement of Mega Sport Event Legacies», Journal of Sport and Tourism, vol. 12, $\mathrm{n}^{\circ}$ 3-4, p. 207-227.

PREUSS, Holger (2007b) «FIFA World Cup 2006 and its Legacy on Tourism ", DANS Trends and Issues in Global Tourism, sous la direction de Roland CONRADY et Martin BUCK, p. 83-103. Berlin : Springer.

RITCHIE, J. R. Brent et Ju YANGZHOU (1987) «The role and impact of mega-events and attractions on national and regional tourism development: a conceptual and methodological overview», DANS Proceedings of the $37^{\text {th }}$ annual congress of the international association of scientific experts in tourism (AIEST), p. 17-58, Calgary.

STRINGER Yvon (1993) «Le mirage des retombées économiques», DANS L'analyse coûts-avantages - Défis et controverses, sous la direction de G. GAUTHIER et M. THIBAULT, p. 335-350. Paris : Economica, coll. «Gestion».

TEED, K.C. (2006) «Mega-events, sport tourism, and the Olympic Games», Tourism Review International, vol. 10, p. 205-206.

U.R.S. FINANCE AND ECONOMICS (2004) Economic Impact of the Rugby World Cup 2003, pour le Département de l'Industrie, du Tourisme et des Ressources, Melbourne.

VOLLET, Dominique et Jean-Paul BOUSSET (2002) «Use of Meta-analysis for the Comparison Transfer of Economics Base Multipliers », Regional Studies, vol. 36, n 5, p. 481-494. 